\title{
A Polling-Based Dynamic Order-Picking System considering Priority Orders
}

\author{
Wenxue Ran, ${ }^{1}$ Sen Liu $\mathbb{D}^{1},{ }^{1}$ and Zhe Zhang $\mathbb{D}^{2}$ \\ ${ }^{1}$ School of Logistics, Yunnan University of Finance and Economics, Kunming 650221, China \\ ${ }^{2}$ School of Economics and Management, Nanjing University of Science \& Technology, Nanjing 210094, China \\ Correspondence should be addressed to Sen Liu; liusencool@163.com
}

Received 21 October 2019; Revised 1 June 2020; Accepted 30 June 2020; Published 24 July 2020

Academic Editor: Roberto Natella

Copyright (c) 2020 Wenxue Ran et al. This is an open access article distributed under the Creative Commons Attribution License, which permits unrestricted use, distribution, and reproduction in any medium, provided the original work is properly cited.

Nowadays, how to offer extremely fast response to customer orders has become a major challenge for warehouse management, especially in e-commerce. Due to the time urgency aspect of some "VIP" orders that need priority processing, one of the most important issues for logistics distribution centres is how to improve the VIP order-picking priority without reducing the common order-picking efficiency. With this consideration, this article put forward a new priority polling model to describe and analyse this problem. We divide orders into priority and common categories according to their time urgency. A mathematical model is established for such a system by applying polling theory, a probability generating function, and an embedded Markov chain. Numerical analysis shows that this priority polling-based picking system can improve the picking efficiency and is well suited to practical operations.

\section{Introduction}

In recent years, China's e-commerce has developed rapidly. According to data from the China International E-commerce Centre, in 2018, consumers spent more than 9 trillion yuan shopping online, and it is expected that the scale of customers preferring to use online platforms will continue to increase in the future. With the growth of e-commerce, customers' expectations for order delivery have also increased; thus, e-commerce firms must have the ability to respond to consumers quickly. JD.com, one of the online platform giants, processes hundreds of thousands of orders every day.

However, the characteristics of e-commerce business, i.e., smaller order sizes and more varieties, put great pressure on logistics centres' order processing activities, which leads to order delivery delays [1]. Therefore, reducing the orderto-delivery time to customers with smaller order sizes and more varieties becomes the primary challenge for distribution centres [2-4]. In general, due to the high operational cost, order picking is considered to be a very important activity in a warehouse, and some studies have even found that the cost of order picking could come to $60 \%$ of the total operational cost [5-7], especially under the environment of e-commerce. In addition, the importance of order sorting must be fully understood in the design of the warehouse [8-10].

Currently, many products are sold both online and offline, and online retailers face difficult challenges of how to organize the logistics supply, both among and after the receipt of order $[4,11]$. First, customers' impulse purchases can lead to the release and cancellation of orders within a certain time horizon [12] which means that the distribution centre could lose opportunity to pick the orders, which will lead to frequent changes in its production plan. Second, firms usually desire to provide rapid and timely delivery in the shortest possible time for the "sudden" late online orders [13]. This is because the Internet makes the probability of customers cancelling orders much higher than in the traditional buying mode since customers can buy another product immediately with a single click, which is much easier than in physical sales [14]. Third, logistics distribution centres have often encountered this type of situation in the e-commerce era. In a batch of orders, a typical order can be 
processed step by step, but some "VIP" orders need to be prioritized. As mentioned above, the frequent cancelling of orders by customers seriously affects the quality of logistics services, which threatens the commitment of logistics providers to clients, especially the owners of VIP orders. Furthermore, the importance of an order is influenced by the customers at different levels. In other words, VIP orders are more important to the e-commence firms than "non-VIP" orders because the loss of VIP customers will lead to more profit losses. Consequently, different types of products require different picking methods.

However, scholars have found that the traditional methods do not deal well with the suddenly appearing VIP orders; Kim et al. [15] introduced a new inventory replenishment method for an automated picking gantry crane, which allowed the late but priority orders to jump the current queue. It was mainly used in short cycle time environments, and they did not consider the fairness of ordinary orders. Hence, polling systems began to be used in picking system studies. Nevertheless, as far as we know, only limited studies have been performed in this area. The pioneering work of Gong and De Koster [11] analysed a dynamic order-picking system based on a polling model, in which the order pickers walk around the picking area to sort out all the backlogged orders in their picking paths. The research results showed that compared with traditional batch-picking methods (as indicated by Le-Duc and de Koster [16]), the proposed polling system-based dynamic order-picking model can effectively bring down the order throughput time. Gong et al. [13] also introduced stochastic variables in a polling system to analyse such picking models and in an approximate way described the complete order waiting time distribution and the accuracy of the polling-based order-picking systems. Claeys et al. [17] designed a polling system in a new service discipline for analysing the order flow time problem of parts-to-picker and used periodic time to deduce the random boundary, which proved to be helpful in setting the target for the storage area throughput.

Nevertheless, the existing polling systems studies on order picking do not consider the situation in regard to priority orders, whereas in a real situation, some VIP orders need to be prioritized over others. Therefore, to solve this problem, we propose a novel combined picking model based on a polling system. We divide the order queues into priority queues (priority orders) and multiple ordinary queues (ordinary orders). Priority orders refer to orders that need to be processed with priority, such as some VIP orders, which need to be processed immediately. Ordinary orders refer to orders that do not need to be processed immediately and have loose time windows. We propose an "exhaustive parallel 1-limited" based dynamic order-picking model, which combines a polling system with the probability generating function approach and the Markov chain to solve this type of picking problem. Due to the service strategy of "exhaustive parallel 1-limited" service strategy, the novel model can treat ordinary orders fairly while improving the picking efficiency of priority orders. The numerical analysis and discussion prove the practicability and scientificity of the method proposed in this article.

The organization of the study is structured as follows. Section 2 gives the literature review. Section 3 introduces the proposed polling system-based picking model. A numerical example is demonstrated in Section 4. Section 5 gives the conclusions and discussion.

\section{Literature Review}

The first related stream of the literature is on the picking activity, which involves stock-to-picker systems, order batching, inventory allocation policies, and order-picking sequencing $[7,8,18-20]$. In a stock-to-picker system, there are three types of order-picking strategies as follows: stockto-picker, picker-to-stock, and automated dispensing systems [21-23]. In storage assignment policies, there are several categories, i.e., random storage, dedicated storage, closest open location storage, family grouping, and classbased storage [24-26]. The idea of order batching involves grouping orders to be picked in a separate route, thereby reducing the theoretical time per pick, with the purpose of determining the best order batching configuration to minimize the distance or travel time [18, 27-29]. Orderpicking sequencing programs focus on the path/route taken when travelling to the pick locations while considering the item extraction order; these apply to warehouses with fully automated picking systems and manual picking operations [30-32]. In recent years, many scholars have realized the significance of mankind factors in order picking, and the study of mankind factors is increasingly gaining popularity [6, 33-37]. For example, Grosse and Glock [38, 39] presented a method to model and discuss the influence of laborer learning, forgetting, and remembering on the storage assignment strategies and order-picking time. However, the methods mentioned in the above studies fail to handle the situations with smaller order sizes and more varieties that are characteristic of e-commerce.

To improve the order-picking efficiency, scholars began using polling systems. A polling system can effectively describe the queue arrival and processing, i.e., a system of multiple queue arrivals in a cycle by a single server, and includes three processes, as follows: queue arrival, serving queue, and switching service object [40]. Much research exists on polling systems, which are used widely in many systems, such as computer and telecommunications networks [1, 41-43]. There are several representative control strategies: gated, exhaustive, limited, $k$-limited, and timelimited. For the gated control strategy, the machine dispenses only the products in series which are turned up when it is checked at a queue [44]. For the exhaustive control strategy, the machine dispenses all products in series in a queue till it is empty. With regard to a limited control strategy, one product in a queue at most is dispensed at a cycle. Under the $k$-limited control strategy, the machine will switch to next queue when $k$ units of products within the current queue have been serviced or no one needs service. However, under a time-limited control strategy, the machine services the current queue according to time rather than the amount of products [45]. Nevertheless, this stream of the literature did not consider the situation in regard to VIP orders, while our paper addresses the issue by trading off the picking service for priority orders and ordinary orders. 


\section{Model of the Proposed Priority Order- Picking System}

3.1. Problem Statement. This study is based on the automatic control strategy of picking equipment in the Baisha logistics distribution centre in Changsha, Hunan Province, China. The Baisha logistics distribution centre is a subsidiary of Hunan Tobacco Company, and its "delivery range" covers Changsha city. At present, most Chinese logistics distribution centres are automating their business processes, and the picking goods are regular unit materials in cartons. In the case of Baisha, for example, the orders are made up of different numbers and brands of unit materials, which are placed by customers through e-commerce and mobile Internet, and the picking delivery time is periodic and punctual. The company promises that the e-commerce orders will be delivered within 24 hours because many retailers are using e-commerce for temporary replenishment.

The traditional order-picking process is to batch large numbers of orders and release them in the workshop. With increasing number of orders, the order batch formation time and order delivery time need to be shortened to ensure that customer demand is met. More efficient picking systems should be studied. Hence, we propose an "exhaustive parallel 1-limited" based dynamic order-picking system that is similar to the system of Gong and De Koster [11], which is described as a "dynamic picking system (DPS)." In a DPS, orders are picked in batches and arrive continuously online, whereafter they are sorted according to client's requirement $[11,18]$. The "exhaustive parallel 1-limited" based DPS can be explained as follows: the picking system is composed of $N$ ordinary picking machines and one priority picking machine. The newly arrived orders include ordinary orders, which have loose time windows, and priority VIP orders, which need to be processed immediately. For example, at a certain moment, the cache holds an ordinary order queue and a priority order queue which are waiting for picking service. When the picking machine starts to work, the priority order queue first begins to accept service until all orders within the queue are served (the exhaustive control strategy); then, the machine turns to pick the ordinary order queue, but only handles one order (the 1-limited control strategy), and then switches to serve a next priority order queue.

Based on the polling system, we divide the picking operation into three processes as follows: the order arriving process, the picking process, and the order polling switch process. First, all three processes are stochastic, and they depend on the consumer's purchase timing, the picker's efficiency, and the picker's adjustment flexibility, respectively. Second, the process of the previous order will obviously affect the queuing time of the next order. These characters illustrate that the picking model satisfies the conditions of memorylessness and conditionally independent, which are required by the Markov chain.

In the polling system, priority orders and ordinary orders are dealt with in parallel operations, with priority orders under an exhaustive control strategy and ordinary orders under a parallel 1 -limited control strategy. Figure 1 represents the studied "exhaustive parallel 1-limited" based order-picking model.

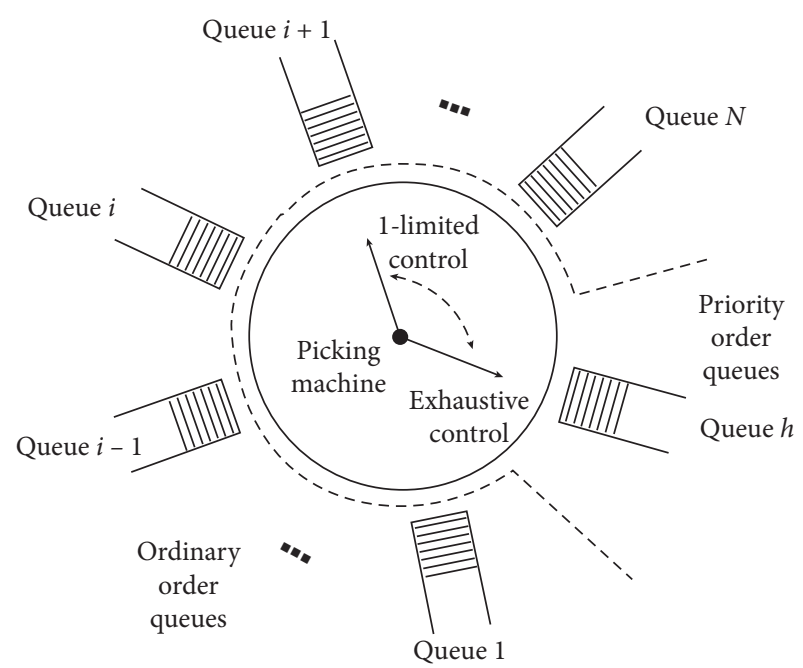

FIGURE 1: The "exhaustive parallel 1-limited" based picking system.

Each arriving order is assumed to have a Poisson distribution; when the picking system begins to work, if ordinary orders need the picking operation, the ordinary picking machines pick them under the 1-limited control strategy (the ordinary order-picking task is not empty). For the picking operation of priority orders, the priority picking machine picks them under an exhaustive control strategy. The exhaustive control strategy reflects the priority of the VIP orders. The 1-limited service policy can judge the arrival situation of the current order queue of the picking machine. With the current order queue arrivals, the picking machine begins to operate. If the current order queue is empty, then the picking machine transfers services to the next order queue. The 1-limited control strategy can then avoid repeated picking and disorder in the ordinary orders.

In addition, there are several basic conditions for the polling system-based picking model as follows:

(1) We assume that one picking machine only can pick one order queue at the same time because a complete order queue includes priority order queues and ordinary order queues. In the mass production environment, the picking system, after finishing a complete order queue, can switch to the next one; otherwise, it will cause disorder in the order queues. Hence, the priority order queues and ordinary order queues cannot be picked at the same time by one picking machine.

(2) The picked goods are all the same kind. The orders are determined by the stochastic needs of the customers, and the quantity of orders is multiples of the unit materials. For example, in this study, one order is composed of different numbers and brands of cigarettes in cartons, and a pack of cigarettes denotes one unit of materials. Each order does not affect each other and has the same probability distribution.

(3) The picking times of the priority order queue and ordinary order queue change with different control strategies. The former is picked by the priority unit in 
the "exhaustive" strategy, while the latter is picked by the ordinary unit in the "1-limited" strategy.

(4) The unit order that enters the order queues is normalized, independent of each other, and follows the same distribution. When one order involves under picking, the order-picking time is independent from the other orders, which also have the same probability distributions. The switch time between contiguous order queues is independent and has the same probability distribution.

(5) The order quantities are not fixed and do not produce information loss.

3.2. Notations and Definitions. Ordinary order queue and priority order queue are simplified to ordinary queue and priority queue, respectively, and all the notations of this paper are as follows:

$\xi_{i}(n)$ : the quantity of unit materials in ordinary queue $i$ $(i=1,2, \ldots, N)$ at moment $t_{n}$.

$\xi_{h}(n)$ : the quantity of unit materials in the priority queue at moment $t_{n}$.

$\xi_{h}\left(n^{*}\right)$ : the quantity of unit materials in the priority queue at moment $t_{n^{*}}$ when the picking machine is shifting from ordinary queue $i$ to the priority queue.

$v_{i}$ : the time that the picking machine is serving ordinary queue $i$.

$v_{h}$ : the time that the picking machine is serving the priority queue.

$u_{i}$ : the time consumed when the picking machine switches from ordinary queue $i$ to the priority queue.

$\eta_{j}\left(\nu_{i}\right)$ : the quantity of unit materials in ordinary order that need to be picked in queue $j$ in $V_{i}$ time $(i=1,2, \ldots$, $N, h)$.

$\eta_{j}\left(\nu_{h}\right)$ : the quantity of unit materials of priority order that need to be picked in queue $j$ in $V_{h}$ time.

$\mu_{j}\left(u_{i}\right)$ : the quantity of unit materials that are switched to queue $j$ in $u_{i}$ time $(i=1,2, \ldots, N, h)$.

$z_{i}$ : the state of ordinary queue $i$.

$z_{h}$ : the state of the priority queue.

$\lambda_{j}$ : the mean time of the arrived $\lambda_{1}=\lambda_{2}=\ldots=\lambda_{N}=\lambda$ ordinary queue $j(j=1,2, \ldots, N)$.

$\lambda_{h}$ : the mean time of the arrived priority queue.

$\beta_{j}$ : the mean time that the picking machine is serving ordinary queue $j(j=1,2, \ldots, N), \beta_{1}=\beta_{2}=\ldots=$ $\beta_{N}=\beta$.

$\beta_{h}$ : the mean time that the picking machine is serving priority the queue.

$r_{j}$ : the switch time between ordinary queue $j(j=1,2$, $\ldots, N)$ and another queue, $r_{1}=r_{2}=\ldots r_{N}=r$.

$A_{j}$ : the probability generating function of the time of the arrived ordinary queue $j$.

$A_{h}$ : the probability generating function of the time of the arrived priority queue.
$B_{j}$ : the probability generating function of the time that the picking machine is serving ordinary queue $j$.

$B_{h}$ : the probability generating function of the time that the picking machine is serving the priority queue.

$R_{i}$ : the probability generating function of the switch time among ordinary queue $i$ and another queue.

$Q$ : the total number of orders.

$Q_{h}\left(z_{h}\right)$ : the probability distribution function of the priority queue's $z_{h}$ state.

$t_{n}$ : the time stamp of the order queue's arrival.

$\varphi$ and $\omega$ : the integer coefficient without practical concern.

$n^{*}$ is contained in a subset of $n$, which denotes the more detailed division of $n$.

$t^{*}$ is contained in a subset of $t$, which denotes the more detailed division of $t$.

Note: the "queue ... switched to ... queue" means that the entire process of an order queue is switched to the next one because there is a time gap between an order queue and the next one when the machine is picking orders.

Let the order arrival process be a Markov stochastic process at moments $t_{n}, t_{n}^{*}$, and $t_{n+1}\left(t_{n}<t_{n}^{*}<t_{n+1}\right)$ with a Poisson distribution and no aftereffect. In practice, the number of order VC queues is relatively determined. Therefore, a discrete-time countable state variable can structure an embedded Markov chain, and the system state variable at the next moment is only related to that at the previous moment. According to the literature [46], the Markov process has the characteristics of nonperiodic and ergodic in a steady state of the system and has a particular steady-state distribution. This means that when the ordinary orders $i(1,2, \ldots, N)$ are being picked at moment $t_{n}, \xi(n)$ ordinary orders are waiting for picking. At this moment, the state variables of the system and their probability generating function are $\left\{\xi_{1}(n), \xi_{2}(n), \cdots, \xi_{N}(n), \xi_{h}(n)\right\}$ and $G_{i}\left(z_{1}, \cdots, z_{N}, z_{h}\right)\left(g_{i}(j)\right.$ is the first-order partial derivative of $G_{i}$ with respect to $z_{j}$ ), respectively. Similarly, the state variables of the system and their probability generating function at moment $t_{n}^{*}$ are $\left\{\xi_{1}\left(n^{*}\right), \xi_{2}\left(n^{*}\right), \cdots, \xi_{N}\left(n^{*}\right)\right.$, $\left.\xi_{h}\left(n^{*}\right)\right\}$ and $G_{i h}\left(z_{1}, \cdots, z_{N}, z_{h}\right)\left(g_{i h}(j)\right.$ is the first-order partial derivative of $G_{i h}$ with respect to $z_{j}$ ), respectively.

\subsection{Probability Generating Function of the Picking System}

3.3.1. Probability Generating Function with Picking Priority Orders. Set the total time for the picking machine to complete the picking services of $k$ orders within priority order queue as $\tau_{k}$ and the time consumed for picking the first order within ordinary order queue as $S_{h, 1}$. Since the priority order queue adopts the exhaustive control strategy, the picking machine needs to serve the orders which are inserted in the $g$-th time slot as well as the orders entered into the cache during the picking operation. The operation time they need is $V_{h, m}=\sum_{1=1}^{\xi_{h}(m)}\left(S_{h, 1}+\sum_{k=1}^{S_{h, 1}} V_{h, k}\right)$. The time axis is divided by unit time slot, and the object of analysis is discrete-time system. First, the picking machine at time $t_{n}$, 
according to the 1-limited service policy, picks the ordinary order queue. Now, the state variables of the DPS are $\left\{\xi_{1}(n), \xi_{2}(n), \cdots, \xi_{N}(n), \xi_{h}(n)\right\}$. At time $t_{n}^{*}$, the ordinary order end picking activity and the picking machine switch to pick the priority order $h$ under an exhaustive control strategy. And the state variables of the DPS are $\left\{\xi_{1}\left(n^{*}\right), \xi_{2}\left(n^{*}\right), \cdots, \xi_{N}\left(n^{*}\right), \xi_{h}\left(n^{*}\right)\right\}$. After picking, the ordinary order queue $i+1$ is polled at time $t_{n+1}$. Now, the system's state variables are $\left\{\xi_{1}(n+1), \xi_{2}(n+1), \cdots\right.$, $\left.\xi_{N}(n+1), \xi_{h}(n+1)\right\}$. Note that the system's state variable at time $t_{n+1}$ is only correlated to that at time $t_{n}^{*}$, which is a Markov process without aftereffect. Thus, we can derive the state transition equation of the picking system as follows:

$$
\begin{aligned}
\xi_{j}\left(n^{*}\right) & = \begin{cases}\xi_{j}(n)+\eta_{j}\left(v_{i}\right), & i \neq j, \\
\xi_{j}(n)+\eta_{j}\left(v_{i}\right)-1, & i=j, \\
\xi_{i}(n) \neq 0,\end{cases} \\
\xi_{j}\left(n^{*}\right) & = \begin{cases}\xi_{j}(n)+\mu_{j}\left(u_{i}\right), & i \neq j, \\
\mu_{j}\left(u_{i}\right), & i=j, \\
\xi_{i}(n)=0 .\end{cases}
\end{aligned}
$$

Referring to the work of Takagi [47], the condition of a stable system operation is given by $N \lambda \beta+N \lambda r+\lambda_{h} \beta_{h}<1$, and $\quad G_{i+1}\left(z_{1}, z_{2}, \ldots, z_{i}, \ldots, z_{N}, z_{h}\right)=$ $\lim _{n \longrightarrow \infty} E\left(\prod_{\substack{j=1 \\ j \neq i}}^{N} z_{j}^{\xi_{j}^{(n+1)}} \cdot z_{h}^{\xi_{h}(n+1)}\right)$ is defined as the quantity of priority queues' probability generating function waiting for picking at moment $t_{n}^{*}$. We have $=\lim _{n \longrightarrow \infty} \sum_{p-0}^{\infty}$ $E\left(\prod_{\substack{j=1 \\ j \neq i}}^{N} z_{j}^{\xi_{j}(n+1)} \cdot z_{h}^{\xi_{h}(n+1)} \mid \xi_{i}(n)=a\right) P\left\{\xi_{i}(n)=a\right\}$, where $P\left\{\xi_{j}\left(n^{*}\right)=z_{j}, j=1,2, \cdots N, h \mid \xi_{j}\left(n^{*}\right)=a\right\}$ denotes the probability distribution of the system state at moment $t_{n}^{*}$. Through simplification, we can gain the probability generating function of the picking machine as follows:

$$
\begin{aligned}
G_{i h}\left(z_{1}, \cdots, z_{N}, z_{h}\right)= & \frac{1}{z_{i}}\left(A_{h}\left(z_{h}\right) \prod_{j=1}^{N} A_{j}\left(z_{j}\right)\right)\left[G_{i}\left(z_{1}, \cdots, z_{N}, z_{h}\right)-G_{i}\left(z_{1}, \cdots, z_{N}, z_{h}\right) \mid z_{i}=0\right] \\
& +R_{i}\left(A_{h}\left(z_{h}\right) \prod_{j=1}^{N} A_{j}\left(z_{j}\right)\right) G_{i}\left(z_{1}, \cdots, z_{N}, z_{h}\right) \mid z_{i}=0 i, j=1,2, \cdots, N, h .
\end{aligned}
$$

\subsubsection{Probability Generating Function in Picking Ordinary} Orders. When the picking machines pick order $i+1$ at moment $t_{n+1}$ under the exhaustive control strategy, the state transition equation of the DPS at moment $t_{n+1}$ is as follows: $\left\{\xi_{j}(n+1)=\xi_{j}\left(n^{*}\right)+\eta_{j}\left(v_{h}\right), i \neq j ; \xi_{j}(n+1)=0, j \neq h\right\}$. Using the same method as Takagi [48], we have

$G_{i+1}\left(z_{1}, z_{2}, \cdots, z_{i}, \cdots, z_{N}, z_{h}\right)=$

$\lim _{n \longrightarrow \infty} E\left(\prod_{\substack{j=1 \\ j \neq i}}^{N} z_{j}^{\xi_{j}(n+1)} \cdot z_{h}^{\xi_{h}(n+1)}\right)$ which denotes the quantity of ordinary queues' probability generating function waiting for picking at moment $t_{n+1}$. Since the orders arrive at each queue and wait in line based on the independent Poisson process, the service time provided by the picking machine for each order is independent from the others, and the system state variables' probability generating function can be formulated as follows:

$$
\begin{aligned}
& G_{i+1}\left(z_{1}, z_{2}, \ldots, z_{N}, z_{h}\right)=\lim _{n \longrightarrow \infty} E\left(\prod_{j=1}^{N} z_{j}^{\xi_{j}(n+1)} \cdot z_{h}^{\xi_{h}(n+1)}\right), \\
& G_{i h}\left(z_{1}, z_{2}, \ldots, z_{N}, B_{h}\left(\prod_{j=1}^{N} A_{j}\left(z_{j}\right) Q_{h} \prod_{j=1}^{N} A_{j}\left(z_{j}\right)\right)\right),
\end{aligned}
$$

where $i=1,2, \ldots, N$.

The random variables of the time require the picking machines serving the orders with the exhaustive control strategy in the priority queue to follow a probability distribution $Q_{h}\left(z_{h}\right)$, which is the same distribution and independent. Then, the probability distribution function can be obtained by calculating

$$
Q_{h}\left(z_{h}\right)=E\left[Z_{h}^{V_{h, m}}\right]=E\left[Z_{h}^{\Gamma} Q_{h}\left(z_{h}\right)^{\Gamma}\right]=E\left[B^{\xi_{h}(m)}\left(z_{h} Q_{h}\left(z_{h}\right)\right)\right]=A_{h}\left(B_{h}\left(z_{h} Q_{h}\left(z_{h}\right)\right)\right),
$$


where $\Gamma=\sum_{=1}^{\xi_{h}(m)} S_{h, 1}$.

$$
\begin{aligned}
Q_{h}\left(z_{h}\right)= & A_{h}\left(B_{h}\left(z_{h} Q_{h}\right)\left(z_{h}\right)\right) ; \\
G_{i h}\left(z_{h}\right)= & R_{i}\left(A_{h}\left(z_{h}\right)\right)\left\{B_{i}\left(A_{h}\left(z_{h}\right)\right)\right. \\
& {\left[1-G_{i}(1,1, \ldots, 0, \ldots, 1,1)\right] } \\
& \left.+G_{i}(1,1, \ldots, 0, \ldots, 1,1)\right\} .
\end{aligned}
$$
The
follows:

The first-order derivative of $Q_{h}\left(z_{h}\right)$ when $z_{h}=1$ is as

$$
\begin{aligned}
Q_{h}^{\prime \prime}(1)= & A_{h}^{\prime \prime}(1)\left(B_{h}^{\prime}(1)\right)^{2}\left(1+Q_{h}(1)\right)^{2}+A_{h}^{\prime}(1) B_{h}^{\prime \prime}(1)\left(1+Q_{h}(1)\right)^{2} \\
& +A_{h}^{\prime}(1) B_{h}^{\prime}(1)\left(2 Q_{h}^{\prime}(1)+Q_{h}^{\prime \prime}(1)\right) .
\end{aligned}
$$

That is,

$$
Q_{h}^{\prime \prime}(1)=A_{h}^{\prime \prime}(1) \beta_{h}^{2}\left(1+Q_{h}(1)\right)^{2}+\lambda_{h} B_{h}^{\prime \prime}(1)\left(1+Q_{h}(1)\right)^{2}+\lambda_{h} \beta_{h}\left(2 Q_{h}^{\prime}(1)+Q_{h}^{\prime \prime}(1)\right) .
$$

Solving the above equation gives the following:

$$
Q_{h}^{\prime \prime}(1)=\frac{1}{\left(1-\lambda_{h} \beta_{h}\right)^{3}}\left[A_{h}^{\prime \prime}(1) \beta_{h}^{2}+B_{h}^{\prime \prime}(1) \lambda_{h}+2 \lambda_{h}^{2} \beta_{h}^{2}\left(1-\lambda_{h} \beta_{h}\right)\right] .
$$

\subsection{The Characteristic Parameters of the System Generating Function Calculation}

3.4.1. Mean Queue Length of Priority Orders (MQLPO). MQLPO refers to the number of orders $g_{i h}(j)$ of queue $j$ at moment $t_{n}^{*}$, i.e., the average amount of the priority queue's orders waiting for service in the buffer. First, by definition, the second-order partial derivatives of $G_{i}(j)$ and $G_{i h}(j)$ are gained, accordingly, as follows:

$$
g_{i}(j, k)=\lim _{z_{1}, z_{2}, \ldots, z_{i}, \ldots, z_{N}, z_{h} \longrightarrow 1} \frac{\partial^{2} G_{i}\left(z_{1}, \ldots, z_{i}, \ldots, z_{N}, z_{h}\right)}{\partial z_{j} \partial z_{k}},
$$

$$
g_{i h}(j, k)=\lim _{z_{1}, z_{2}, \ldots, z_{i}, \ldots, z_{N}, z_{h} \longrightarrow 1} \frac{\partial^{2} G_{i h}\left(z_{1}, \ldots, z_{i}, \ldots, z_{N}, z_{h}\right)}{\partial z_{j} \partial z_{k}} .
$$

Plugging equations (1), (2), (10), and (12), we have the following equation:

$$
\begin{aligned}
g_{i+1}(i, i)= & g_{i h}(i, i)+2 \lambda \beta_{h}\left(1+Q_{h}^{\prime}(1)\right) g_{i h}(i, h)+\lambda^{2} \beta_{h}^{2}\left(1+Q_{h}^{\prime}(1)\right) g_{i h}(h, h) \\
& +\left(\lambda^{2} B_{h}^{\prime \prime}(1)\left(1+Q_{h}^{\prime}(1)\right)^{2}+\beta_{h}\left(A^{\prime \prime}(1)+\left(2 \lambda^{2}+A^{\prime \prime}(1)\right) Q_{h}^{\prime}(1)+A^{\prime \prime}(1) Q_{h}^{\prime \prime}(1)\right)\right) g_{i h}(h),
\end{aligned}
$$

where $g_{i+1}(k, h)=0$ and $g_{i+1}(i, h)=0$. Then, we can obtain the MQLPO as follows:

$$
g_{i h}(h, h)=\frac{N r \lambda}{1-\lambda_{h} \beta_{h}-N(\lambda \beta-\lambda r)}\left[A_{h}^{\prime \prime}(1)(\beta-r)+\lambda_{h}^{2}\left(B^{\prime \prime}(1)-R^{\prime \prime}(1)\right]+\lambda_{h}^{2} R^{\prime \prime}(1)+r A_{h}^{\prime \prime}(1) .\right.
$$

3.4.2. Mean Cyclic Period (MCP). The MCP refers to the statistical average time of the $N+1$ order to complete a picking action, which consists of the picking time and polling time. In this model, when the queue buffer is not entirely empty, the query conversion time can be saved, so a small mean cyclic period (MCP) can be obtained. According to the generating function of system state probability, we can obtain the following equation based 
on the relationship of the probability generating function:

$$
\begin{aligned}
i= & 1,2, \ldots, N, h ; \\
j & =1,2, \ldots, N, h, \\
1-G_{i 0}= & 1-\left.G\left(1, \cdots, z_{i}, 1, \cdots, 1\right)\right|_{z_{t}=0} \\
& =\frac{\lambda_{i} \sum_{i=1}^{N} r_{i}}{1-\lambda_{h} \beta_{h}-\left(\sum_{i=1}^{N} \lambda_{i} \beta_{i}-\sum_{i=1}^{N} \lambda_{i} r_{i}\right)}, \\
1-G_{i o}= & \lambda_{i} \bar{\theta} .
\end{aligned}
$$

Therefore, the MCP can be formulated as follows:

$\bar{\theta}=\frac{\left(n_{1}+n_{2}\right) \gamma}{1-\left(n_{1}+n_{2}\right) \rho_{i}-\rho_{h}}, \quad \rho_{i}=\lambda_{i} \beta_{i} ; \rho_{h}=\lambda_{h} \beta_{h} ; i=1,2, \cdots,\left(n_{1}+n_{2}\right)$.

3.4.3. System Throughput (ST). The ST refers to the number of finished orders in a unit time. The ST reflects the theoretical picking capability of the picking machine and can be expressed as follows:

$$
O=N \lambda(\beta-r)+\lambda_{h} \beta_{h}
$$

3.4.4. Mean Queue Length of Ordinary Orders (MQLOO). MQLOO refers to the mean queue length of the ordinary orders. To determine the MQLOO, first we can represent the generating function's first-order partial derivatives as follows:

$$
\begin{array}{r}
g_{i}(j)=\lim _{z_{1}, z_{2}, \cdots, z_{N}, z_{h} \longrightarrow 1} \frac{\partial G_{i}\left(z_{1}, z_{2}, \cdots, z_{N}, z_{h}\right)}{\partial z_{j}}, \\
i=1,2, \cdots, N ; \\
j=1,2, \cdots, N, h .
\end{array}
$$

Plugging equations (1) and (2) into (17), we have the following equation:

$$
\begin{aligned}
g_{i}(k)= & g_{i}(i)+(i-k)\{\lambda r+(\lambda \beta-\lambda r) \\
& {\left[1-G_{i}\left(z_{1}, \cdots, z_{i}, \cdots, z_{N}, z_{h}\right) \mid z_{i}=0\right] } \\
& \left.+\lambda \beta_{h}\left(1+Q_{h}^{\prime}(1)\right) g_{i h}(h)\right\} \\
& -\left[1-\left.G_{i}\left(z_{1}, \cdots, z_{i}, \cdots, z_{N}, z_{j}\right)\right|_{z_{i}=0}\right],
\end{aligned}
$$

where $g_{i h}(h)=N r \lambda_{h}\left(1-\lambda_{h} \beta_{h}\right) / 1-\lambda_{h} \beta_{h}-N(\lambda \beta-\lambda r)$, and $g_{i}(h)$ and $1-G_{i}\left(z_{1}, \cdots, z_{N}, z_{h}\right) \mid z_{i}=0$ can be obtained by calculating $\sum_{j=1}^{N} g_{j+1}(k)$. Then, we can then formulate MQLOO as follows:

$$
\begin{aligned}
g_{i}(i)= & \frac{1-\lambda_{h} \beta_{h}}{2\left(1-\lambda_{h} \beta_{h}-N \lambda \beta\right)} \\
& \times N r\left[1+\frac{\lambda_{h}^{2} \beta_{h}^{2}}{1-\lambda_{h} \beta_{h}}+\frac{\lambda}{1-\lambda_{h} \beta_{h}-N \lambda(\beta-r)}(N(\beta-r))+\frac{\lambda \beta_{h}^{2}\left(\lambda_{h} \beta_{h}+N \lambda(\beta-r)\right)}{\left(1-\lambda_{h} \beta_{h}\right)^{2}}\right] A^{\prime \prime}(1) \\
& +\frac{N^{2} \lambda^{3} r}{\left(1-\lambda_{h} \beta_{h}-N \lambda(\beta-r)\right)\left(1-\lambda_{h} \beta_{h}\right)^{2}} B^{\prime \prime}(1)+\frac{N \lambda^{2} \lambda_{h} \beta_{h}^{2} r}{\left(1-\lambda_{h} \beta_{h}-N \lambda(\beta-r)\right)\left(1-\lambda_{h} \beta_{h}\right)^{2}} B_{h}^{\prime \prime}(1) \\
& +\frac{N r \lambda^{2} \lambda_{h} \beta_{h}}{1-\lambda_{h} \beta_{h}}+\frac{N \lambda^{2}}{1-\lambda_{h} \beta_{h}}\left[1+\lambda_{h} \beta_{h}-\lambda_{h}^{2} \beta_{h}^{2}-\frac{N \lambda r\left(3-2 \lambda_{h} \beta_{h}\right)}{\left(1-\lambda_{h} \beta_{h}-N \lambda(\beta-r)\right)\left(1-\lambda_{h} \beta\right)}\right] R^{\prime \prime}(1) \\
& +\frac{N \lambda\left(2 r\left(1-\lambda \beta-\lambda_{h} \beta_{h}\right)+2 N r \lambda^{2} \lambda_{h} \beta_{h}(\beta-r)+r \lambda \lambda_{h} \beta_{h}\left(1+\lambda_{h} \beta_{h}\right)+(N-1)(r \lambda-2 r \lambda \beta)\right)}{\left(1-\lambda_{h} \beta_{h}-N \lambda(\beta-r)\right)\left(1-\lambda_{h} \beta\right)} .
\end{aligned}
$$

3.4.5. Mean Waiting Time of Priority Orders (MWTPO) and Mean Waiting Time of Ordinary Orders (MWTOO). MWTPO denotes the priority orders' mean waiting time, and MWTOO denotes the ordinary orders' mean waiting time. Let $w_{j}$ be the waiting time between orders $j(j=1,2, \ldots, N, h)$ being picked over those to be sent out, and let $W_{j}\left(z_{j}\right)$ be the probability generating function; $E\left(w_{h}\right)$ and $E\left(w_{i}\right)$ are the average waiting time delays of the customers' priority queues and ordinary queues, respectively.

Then, by using the same solving method of the MWT as in Takagi [48], $W_{i}\left(A\left(z_{i}\right)\right) B\left(A\left(z_{i}\right)\right)=Q_{i}\left(1,1, \cdots, z_{i}\right.$, $1, \cdots, 1)$ exists. Then, we have the derivation $E\left(w_{k}\right)=g_{k}(i) / \lambda T_{C}-1 / \lambda$, which refers to the average time which denotes the time interval between an order arriving at 
the queue $i$ and finishing its picking service. Furthermore, we can obtain $E\left(w_{s}\right)=A^{\prime \prime}(1) /\left(2 \lambda^{2}\right)$, which denotes the difference among the order VC queue's arrival time and the unit time interval. According to $E\left(w_{i}\right)=E\left(w_{k}\right)-E\left(w_{s}\right)$, we can calculate MWTOO as follows:

$$
\begin{aligned}
E\left(w_{i}\right)= & \frac{g_{i}(i)}{\lambda T_{C}}-\frac{A^{\prime \prime}(1)}{2 \lambda^{2}}-\frac{1}{\lambda}=\frac{1-\lambda_{h} \beta_{h}-N \lambda(\beta-r)}{N \lambda^{2} r} \\
& \times g_{i}(i)-\frac{A^{\prime \prime}(1)}{2 \lambda^{2}}-\frac{1}{\lambda} .
\end{aligned}
$$

By using the same MWT solving method as in Takagi [47], we first calculate the mean picking time of order arriving between moment $t_{n}$ and $t_{m}$, as follows: $E\left(w_{h, 1}\right)=$ $g_{i h}(h, h) / 2 \lambda_{h}\left(1+\lambda_{h} \beta_{h}\right) g_{i h}(h)+\lambda_{h} B_{h}^{\prime \prime}(1) / 2\left(1-\lambda_{h}^{2} \beta_{h}^{2}\right)-(1-$ $\left.\lambda_{h} \beta_{h}-\lambda_{h}^{2} \beta_{h}^{2}\right) A_{h}^{\prime \prime}(1) / 2 \lambda_{h}^{2}\left(1+\lambda_{h}^{2} \beta_{h}^{2}\right)$. Then, we calculate the mean delivery time of order arriving between moment $t_{m}$ and $t_{o}$, as follows: $E\left(w_{h, 2}\right)=\lambda_{h} \beta_{h} g_{i h}(h, h) /$ $2 \lambda_{h}\left(1+\lambda_{h} \beta_{h}\right) g_{i h}(h)+\lambda_{h}^{3} \beta_{h}^{2} B_{h}^{\prime \prime}(1) / 2\left(1-\lambda_{h}^{3} \beta_{h}^{2}\right)-\lambda_{h}^{3} \beta_{h}^{2} A_{h}^{\prime \prime}(1) /$ $2 \lambda_{h}^{2}\left(1-\lambda_{h}^{3} \beta_{h}^{2}\right)$. According to $E\left(w_{h}\right)=E\left(w_{h, 1}\right)+E\left(w_{h, 2}\right)$, we can calculate MWTPO as follows:

$$
\begin{aligned}
E\left(w_{h}\right)= & \frac{g_{i h}(h, h)}{2 \lambda_{h} g_{i h}(h)}+\frac{\lambda_{h} B_{h}^{\prime \prime}(1)}{2\left(1-\lambda_{h} \beta_{h}\right)}-\frac{A_{h}^{\prime \prime}(1)}{2 \lambda_{h}^{2}\left(1+\lambda_{h} \beta_{h}\right)}=\frac{\lambda\left(A_{h}^{\prime \prime}(1)(\beta-r)\right)+\lambda_{h}^{2}\left(B^{\prime}(1)-R^{\prime}(1)\right)}{2 \lambda_{h}^{2}\left(1-\lambda_{h} \beta_{h}\right)} \\
& +\frac{\left(1-\lambda_{h} \beta_{h}-(N \lambda \beta-N r \lambda)\right)\left(\lambda_{h}^{2} R^{\prime}(1)+r A^{\prime}(1)\right)}{2 N r \lambda_{h}^{2}\left(1-\lambda_{h} \beta_{h}\right)}+\frac{\lambda_{h} B_{h}^{\prime}(1)}{2\left(1-\lambda_{h} \beta_{h}\right)}-\frac{A_{h}^{\prime \prime}(1)}{2 \lambda_{h}\left(1+\lambda_{h} \beta_{h}\right)} .
\end{aligned}
$$

\section{Numerical Example and Discussion}

4.1. Numerical Example. This section describes how to use the proposed polling systems in actual practice. To facilitate the numerical analysis, we use the order-picking operation of a logistics distribution centre as an example to illustrate the validity of the method.

There are some instructions for the numerical example, which are as follows: all order queues consist of two group orders of the same product, as shown in Table 1, where one group order is in the priority order queue and the other is in the ordinary order queue, as in an actual situation. The outer package of the unit material has a length of $290 \mathrm{~mm}$, a width of $98 \mathrm{~mm}$, and a height of $50 \mathrm{~mm}$, and the order conveyor speed is $60 \mathrm{~m} / \mathrm{min}$; the picking speed per unit material is 3 seconds, the order switch time is 1.2 minutes, and the number of picking stations is 10 . The computing process uses the same normalized parameters, which are $\beta_{\mathrm{h}}=\beta_{i}=\beta$, $\lambda_{\mathrm{h}}=\lambda_{i}=\lambda$, and $\gamma=\gamma_{i}$.

Table 1 provides the actual order structure data, supported by the Baisha logistics distribution centre. When the system is stable, then based on the "exhaustive parallel 1limited" control strategy, we can calculate the characteristic parameters of the system generating function.

Table 2 shows the comparison of the numerical results using the data of Table 1, and we can find that the theoretical values are in close proximity to the actual values from the logistics distribution centre, thus indicating that the proposed "exhaustive and 1-limited" based polling model is valid.

Next, we use the proposed "exhaustive parallel 1-limited" based picking system for further numerical analysis, and the simulation results (Figures 2-6) and analysis are as follows.

Figure 2 shows the change trend between MQLPO and $\lambda$, wherein Figure 2(a) is based on the change in the priority orders' queue quantity $N$, Figure 2 (b) is based on the change in the picking time $(\beta)$ of the priority orders' queue, and Figure 2(c) is based on the change in the switching time $(r)$ between priority orders' queue and the other. As shown in Figure 2, when the other variables are fixed, the MQLPO of the order queue with larger variable $(N / \beta / r)$ increases sharply with the $\lambda$ 's growth compared to the other queues. In addition, if $\lambda$ exceeds a certain threshold $\left(\lambda \times 10^{-3}=18\right.$ in (c)), the diversity of $r$ does not cause a significant difference in the MQLPO between the order queues.

Figure 3 shows the variation trend between MQLOO and $\lambda$, wherein Figure 3(a) is based on the change in the ordinary orders' queue quantity $N$, Figure $3(\mathrm{~b})$ is based on the change in the picking time of $(\beta)$ the ordinary orders' queue, and Figure $3(c)$ is based on the change in the switching time between the ordinary orders' queue and another queue $(\gamma)$. As shown in Figure 3, when the other variables are fixed, the MQLOO of the order queue with a larger variable $(N / \beta / r)$ increases sharply with the $\lambda$ 's growth compared to the other queues.

Comparing Figures 2 and 3, we find that with the increase in the variables $(N, \beta, r)$, the curves of MQLOO $(\lambda)$ and MQLPO $(\lambda)$ all become steeper, i.e., the curves of $\operatorname{MQLOO}(\lambda)$ and MQLPO $(\lambda)$ with the larger variables $(N /$ $\beta / r)$ have greater slopes as $\lambda$ increases. Through further analysis, we also find that in the process of increasing $\lambda$, the MQLPO and MQLOO continue to grow, but the MQLPO maintains a small increase and steady change, and the MQLOO has a sudden increase, especially when the queues' quantity is relatively large. This finding reveals that the MQL in ordinary orders is more sensitive to changes in $\lambda$ compared to priority orders.

Figure 4 reveals the variation trend between MCP and $\lambda$, wherein Figure 4(a) is based on the change in the priority orders' queue quantity $N$, Figure $4(\mathrm{~b})$ is based on the change in the picking time $(\beta)$ of the priority orders' queue, and Figure $4(\mathrm{c})$ is based on the change in the switching time of 
TABLE 1: Product order data.

\begin{tabular}{|c|c|c|c|c|c|c|c|c|c|c|c|c|c|c|c|}
\hline The order number & 1 & 2 & 3 & 4 & 5 & 6 & 7 & 8 & 9 & 10 & 11 & 12 & 13 & 14 & 15 \\
\hline Total quantity of unit materials & 16 & 21 & 15 & 21 & 18 & 10 & 3 & 9 & 13 & 21 & 18 & 1 & 1 & 24 & 24 \\
\hline Number of priority unit materials & 13 & 17 & 8 & 16 & 10 & 9 & 1 & 3 & 4 & 21 & 0 & 0 & 1 & 24 & 0 \\
\hline Number of ordinary unit materials & 3 & 4 & 7 & 5 & 8 & 1 & 2 & 6 & 9 & 0 & 18 & 1 & 0 & 0 & \\
\hline
\end{tabular}

TABLE 2: Comparison of the numerical results under different order structures.

\begin{tabular}{|c|c|c|c|c|c|c|c|c|c|c|}
\hline \multirow{2}{*}{ The order number } & \multicolumn{2}{|c|}{ MCP } & \multicolumn{2}{|c|}{ MQLPO } & \multicolumn{2}{|c|}{ MQLOO } & \multicolumn{2}{|c|}{ MWTPO } & \multicolumn{2}{|c|}{ MWTOO } \\
\hline & $\mathrm{AV}$ & TV & $\mathrm{AV}$ & TV & $\mathrm{AV}$ & TV & $\mathrm{AV}$ & TV & $\mathrm{AV}$ & TV \\
\hline 1 & 14.9308 & 14.9266 & 0.0074 & 0.0075 & 0.3998 & 0.4001 & 0.4577 & 0.4270 & 10.3342 & 10.4502 \\
\hline 2 & 14.2161 & 14.2163 & 0.0094 & 0.0095 & 0.5371 & 0.5371 & 0.4184 & 0.3856 & 11.1207 & 11.2662 \\
\hline 3 & 16.2814 & 16.2764 & 0.0187 & 0.0189 & 0.2466 & 0.2470 & 0.4836 & 0.4697 & 9.8017 & 9.8624 \\
\hline 4 & 14.3752 & 17.3765 & 0.0119 & 0.0120 & 0.5010 & 0.5013 & 0.4289 & 0.3963 & 10.9112 & 11.0366 \\
\hline 5 & 15.6882 & 15.6886 & 0.0206 & 0.0208 & 0.3075 & 0.3078 & 0.4718 & 0.4544 & 9.9691 & 10.0731 \\
\hline 6 & 15.9315 & 15.9312 & 0.0026 & 0.0027 & 0.2763 & 0.2763 & 0.4766 & 0.4621 & 9.8501 & 9.9272 \\
\hline 7 & 19.3682 & 19.3693 & 0.0064 & 0.0065 & 0.0329 & 0.0328 & 0.5009 & 0.4995 & 9.5070 & 9.5072 \\
\hline 8 & 18.2929 & 18.2971 & 0.0181 & 0.0183 & 0.0961 & 0.0961 & 0.4957 & 0.4955 & 9.5733 & 9.6552 \\
\hline 9 & 17.8533 & 17.8559 & 0.0261 & 0.0267 & 0.1272 & 0.1271 & 0.4978 & 0.4924 & 9.6154 & 9.6266 \\
\hline 10 & 13.7259 & 13.7226 & 0 & 0 & 0.6981 & 0.6987 & $\mathrm{NaN}$ & $\mathrm{NaN}$ & 12.2891 & 12.4929 \\
\hline 11 & 20.3635 & 20.3629 & 0.0583 & 0.0600 & 0 & 0 & 0.5052 & 0.5054 & $\mathrm{NaN}$ & $\mathrm{NaN}$ \\
\hline 12 & 20.0011 & 20.0011 & 0.0033 & 0.0033 & 0 & 0 & 0.4989 & 0.5000 & $\mathrm{NaN}$ & $\mathrm{NaN}$ \\
\hline 13 & 19.3662 & 19.3651 & 0 & 0 & 0.0328 & 0.0328 & $\mathrm{NaN}$ & $\mathrm{NaN}$ & 9.4913 & 9.5050 \\
\hline 14 & 13.4806 & 13.4848 & 0 & 0 & 0.8498 & 0.8491 & $\mathrm{NaN}$ & $\mathrm{NaN}$ & 13.5216 & 13.8569 \\
\hline 15 & 20.6532 & 20.6543 & 0.0767 & 0.0800 & 0 & 0 & 0.5130 & 0.5130 & $\mathrm{NaN}$ & $\mathrm{NaN}$ \\
\hline
\end{tabular}

$\mathrm{AV}=$ actual value; $\mathrm{TV}=$ theoretical value.

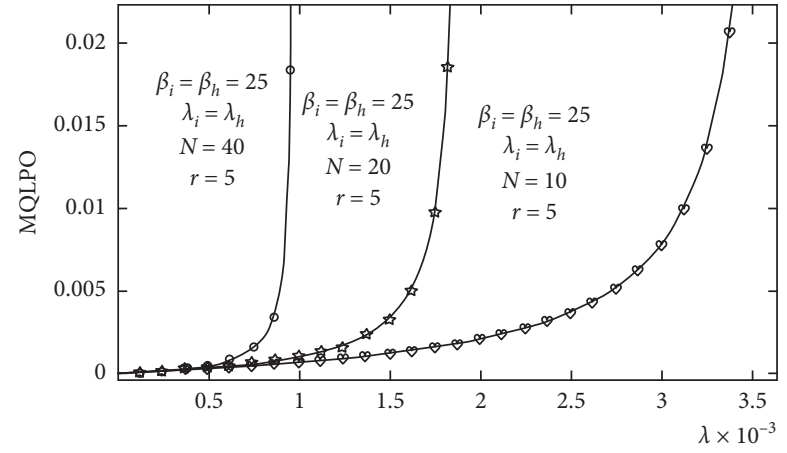

(a)

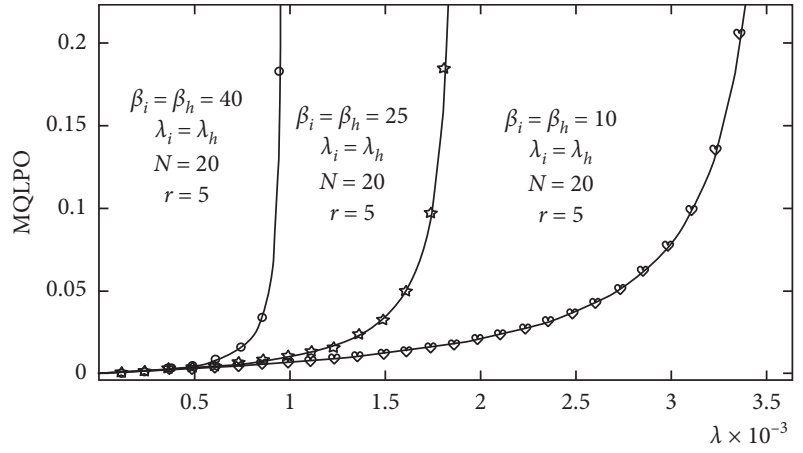

(b)

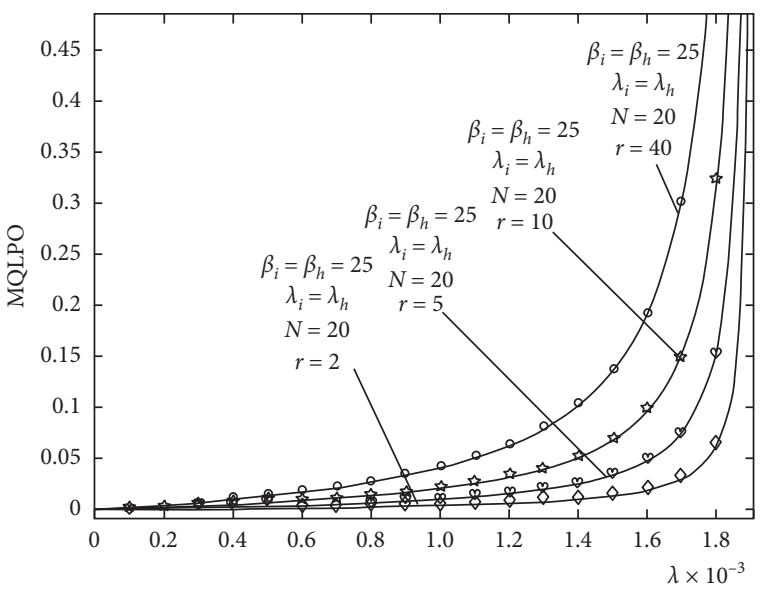

(c)

Figure 2: The change trend of MQLPO. (a) Range of MQLPO with $\lambda^{\prime}$ s change under different $N$. (b) Range of MQLPO with $\lambda^{\prime}$ s change under different $\beta$. (c) Range of MQLPO with $\lambda^{\prime}$ s change under different $r$. 


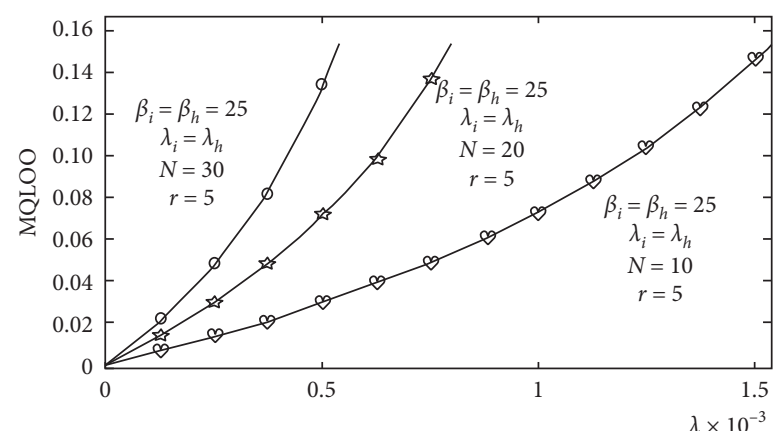

(a)

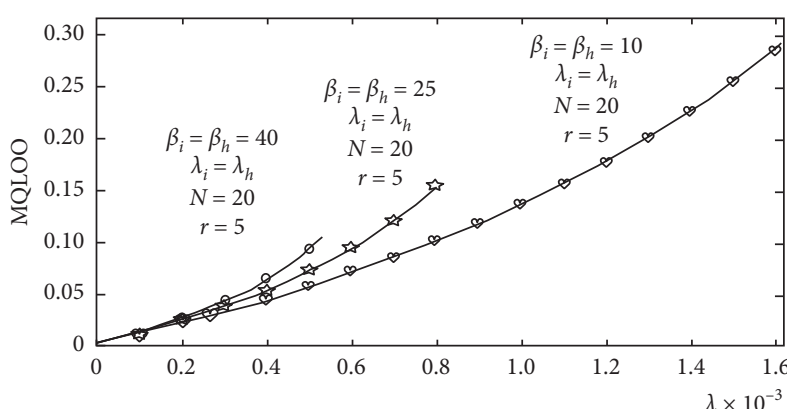

(b)

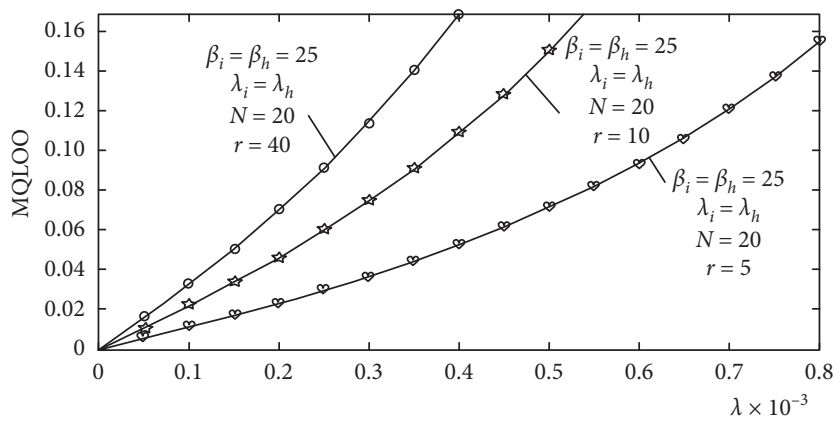

(c)

FIGURE 3: The change trend of the MQLOO. (a) Range of MQLOO with $\lambda^{\prime}$ s change under different $N$. (b) Range of MQLOO with $\lambda^{\prime}$ s change under different $\beta$. (c) Range of MQLOO with $\lambda^{\prime}$ s change under different $r$.

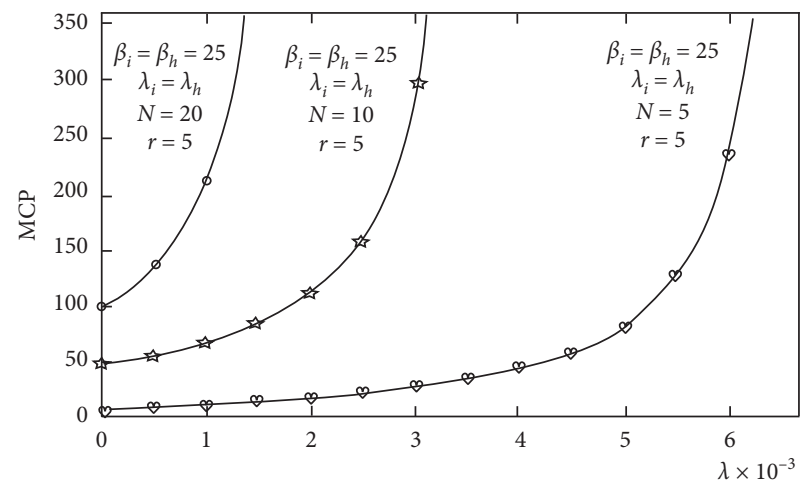

(a)

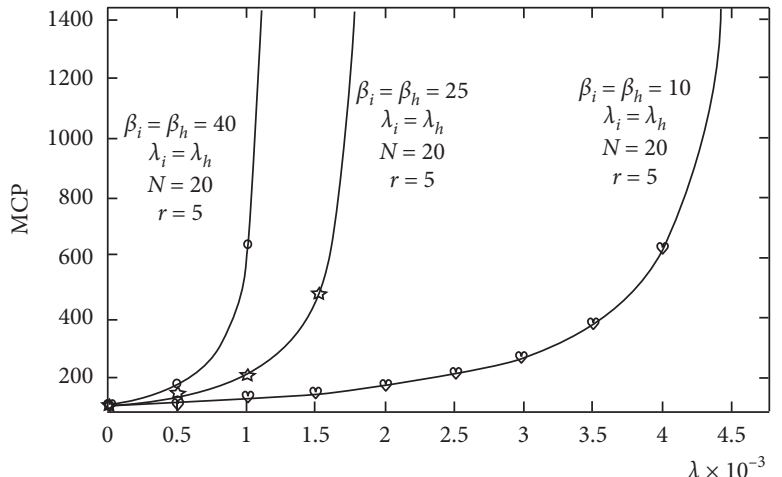

(b)

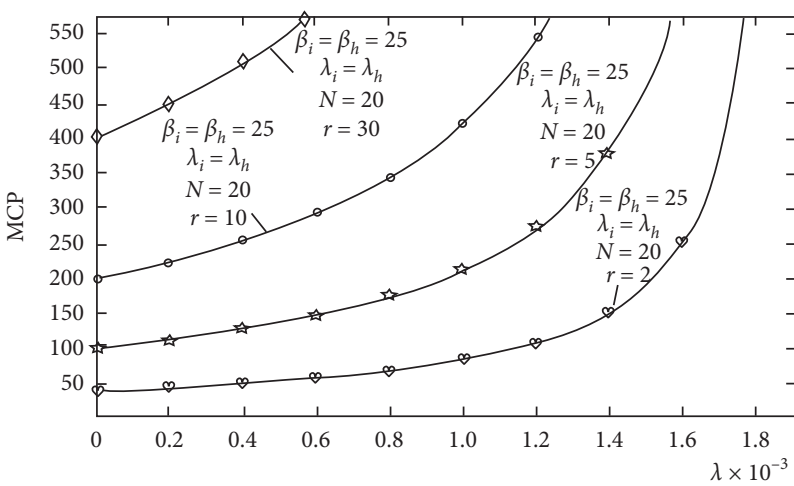

(c)

Figure 4: The change trend of MCP. (a) Range of MCP with $\lambda^{\prime}$ s change under different $N$. (b) Range of MCP with $\lambda^{\prime}$ s change under different $\beta$. (c) Range of MCP with $\lambda^{\prime}$ s change under different $r$. 
the priority orders queue with different $\gamma$. As shown in Figure 4, when the other variables are fixed, the MCP of the order queue with the larger variable $(N / \beta / r)$ increases sharply with the $\lambda$ 's growth compared to the other queues.

Figure 5 represents the variation trend between MWTPO and $\lambda$, wherein Figure 5(a) is based on the change in the priority orders' queue quantity $N$, Figure $5(\mathrm{~b})$ is based on the change in the picking time $(\beta)$ of the priority orders' queue, and Figure 5(c) is based on the change in the switching time between the priority orders' queue and other queues $(r)$. We can see from Figure 5 that under a stable system condition, the MWTPO increases in line with the value of the variable $(N / \beta / r)$. In addition, when the other variables are fixed, the MWTPO of the order queue with a larger variable $(N / \beta / r)$ increases sharply with $\lambda$ 's growth compared to the other queues.

Figure 6 demonstrates the variation trend between MWTOO and $\lambda$, wherein Figure 6(a) is based on the change in the ordinary orders' queue quantity $N$, Figure $6(\mathrm{~b})$ is based on the change in the picking time $(\beta)$ of the ordinary orders' queue, and Figure 6(c) is based on the change in the switching time between the ordinary orders' queue and the other queues $(r)$.We can see from Figure 6 that under a stable system condition, the MWTOO increases in line with the value of the variable $(N / \beta / r)$. Furthermore, when the other variables are fixed, the MWTOO of the order queue with a larger variable $(N / \beta / r)$ increases sharply with the $\lambda$ 's growth compared to the other queues.

In Figures 5 and 6, we can see that with the increase of the variable $(N / \beta / r)$, the curves of MWTOO $(\lambda)$ and MWTPO $(\lambda)$ all become steeper. For example, from Figures 5(a) and 6(a), we can find that keeping all other variables constant, when $N$ increases from 10 to 20 , the curves of MWTPO as well as MWTOO become steeper. This result means that the MWTPO and MWTOO will grow fast with $\lambda$ 's increase under a large variable $(N / \beta / r)$. Through further analysis, we also find that in the process of increasing $\lambda$, the MWTPO and MWTOO continue to grow, but MWTPO maintains a small increase and steady change, and MWTOO suddenly increases (compared to the curve of MWTPO $(\lambda)$, the curve of MWTOO $(\lambda)$ has a greater slope as $\lambda$ is increasing).

From Figures 2 to 6 , we can find that with the increase of the variable $(N / \beta / r)$, the curves of MQLOO $(\lambda), \operatorname{MQLPO}(\lambda)$, $\operatorname{MCP}(\lambda)$, MWTOO $(\lambda)$, and MWTPO $(\lambda)$ all become steeper. In addition, under a stable system condition, the MQLOO, MCP, and MWTOO of the ordinary picking stations increase nonlinearly with increasing station numbers and arrived orders; the MQLPO, MCP, and MWTPO of the priority picking stations decrease slightly. Through the above analysis, we find that the fewer the number of queues is, the smaller the MCP is, and the whole dynamic orderpicking system is more stable and fast-responding.

4.2. Discussion. According to the numerical results, we find the below conclusions:

(1) The numerical examples show that compared with the other polling system conditions, such as
$N \lambda \beta+N \lambda r<1, \quad N \lambda \beta+\lambda r<1, \quad N \lambda \beta<1, \quad$ and $N \lambda \beta+\lambda_{h} \beta_{h}<1$, the new model under the "exhaustive and 1-limited" service strategy system has a more stable performance for the condition $N \lambda \beta+N \lambda r+\lambda_{h} \beta_{h}<1$. Therefore, stable operation of the proposed picking system needs to meet certain conditions, and distribution centre managers should reduce the picking time of one order and increase the picker's efficiency of switching objects for decreasing the system load.

(2) From the nature of the "exhaustive and 1-limited" polling system, we can find in a complete polling picking operation cycle of $N+1$ queues, the priority order queues had $N$ opportunities to be picked based on the "exhaustive" strategy, and each ordinary order queue had only one opportunity to be picked based on the "1-limited" strategy. Therefore, this makes the varieties of MQLPO and MWTPO far less than the varieties of MQLOO and MWTOO, which proves that the proposed picking system can ensure the picking priority of VIP orders to gain higher picking efficiency, while the $N$ ordinary order queues only have one opportunity to be picked in each polling cycle, and this can avoid repeated picking and disorder in ordinary orders. Thus, the proposed picking system has a good picking ability.

(3) The analysis of Figures 5 and 6 shows that increasing the variables $(N / \beta / r)$ will extend the MWT and MQL of the priority orders and common orders. This result suggests that the distribution centre should consider the time of order picking and the speed of picking machine switching between the picking objects, especially the number of picking stations. A large number of picking stations can provide more sorting channels for a variety of order queues; however, more picking stations will result in a rise of the mean waiting time towards the two types of orders. In addition, compared to ordinary order queues, the MWT of the priority order queues has less increases with the growth of $\lambda$. Therefore, the dynamic order-picking system has better robustness for priority orders. This suggests that the novel model can resolve a good deal of priority orders emerging in a short time while maintaining a stable order waiting time. This advantage can help e-commerce companies keep running during the peak periods of online shopping, such as China's "Singles Day" and "Black Friday" in the United States.

(4) The ordinary order-picking system has fair service features, which is particularly important for a good picking system to trade off the difference of picking service between ordinary orders. Although an ordinary order queue has only one opportunity to be picked in Nordinary order queue query cycles, the "1-limited" strategy is adequate to guarantee the equity of the ordinary order-picking service. Thus, the proposed picking system has better fairness, 


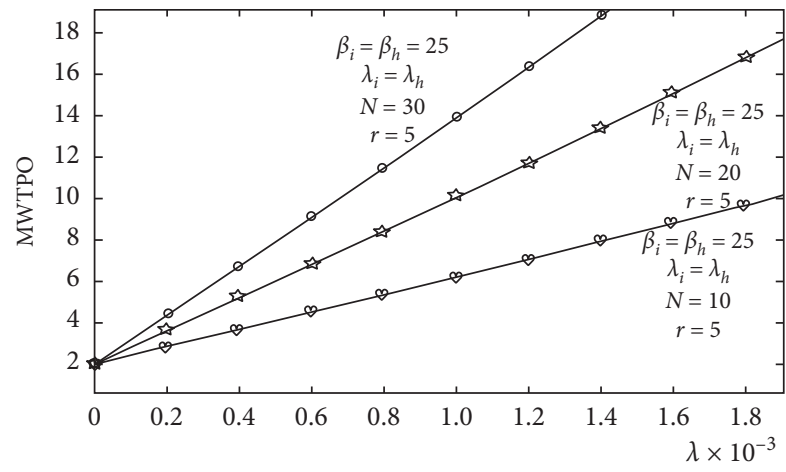

(a)

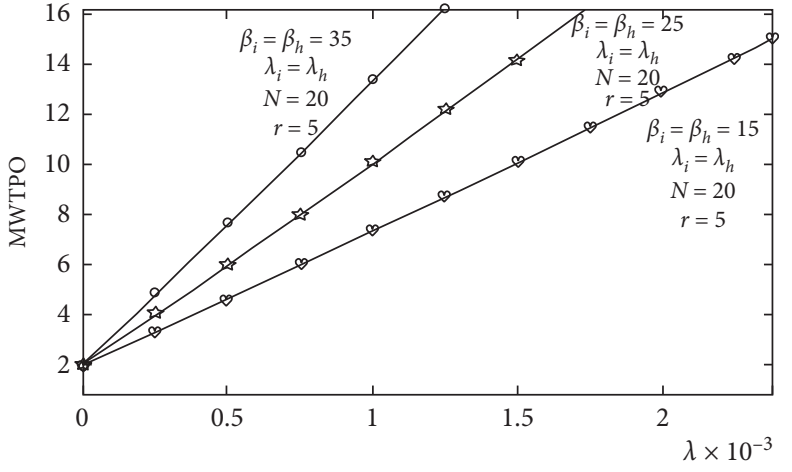

(b)

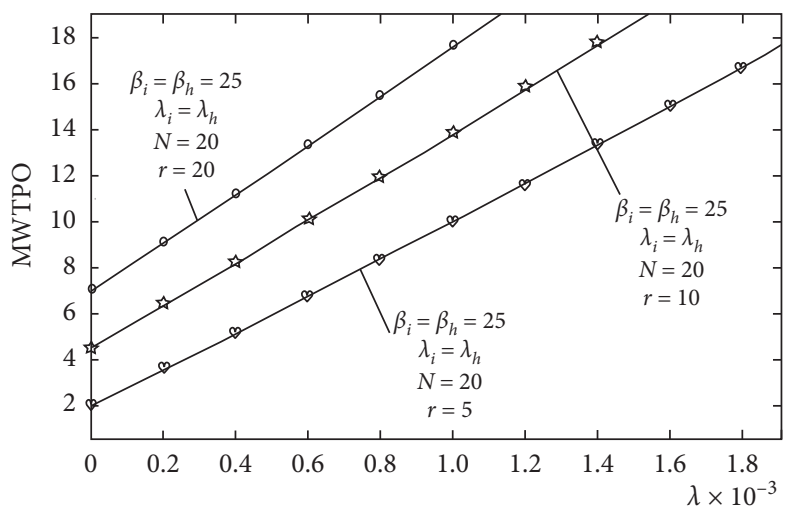

(c)

Figure 5: The change trend of MWTPO. (a) Range of MWTPO with $\lambda^{\prime}$ s change under different $N$. (b) Range of MWTPO with $\lambda^{\prime}$ s change under different $\beta$. (c) Range of MWTPO with $\lambda^{\prime}$ s change under different $r$.

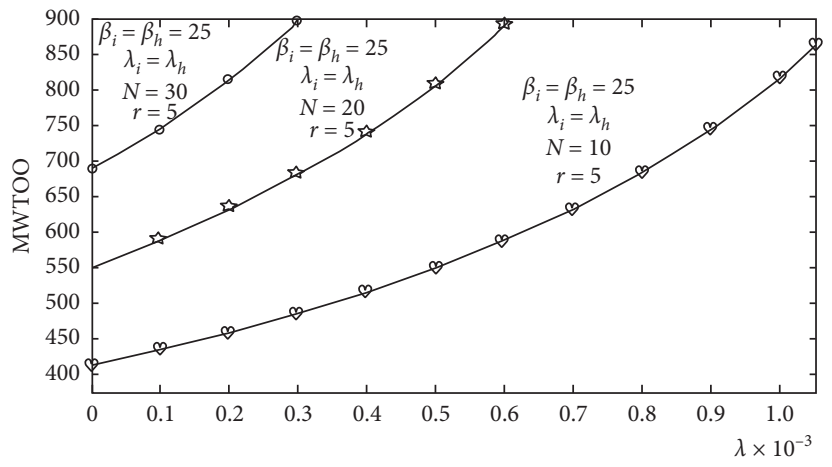

(a)

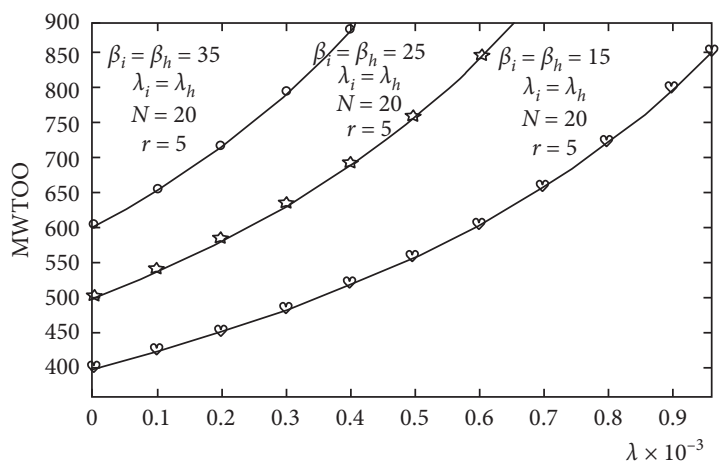

(b)

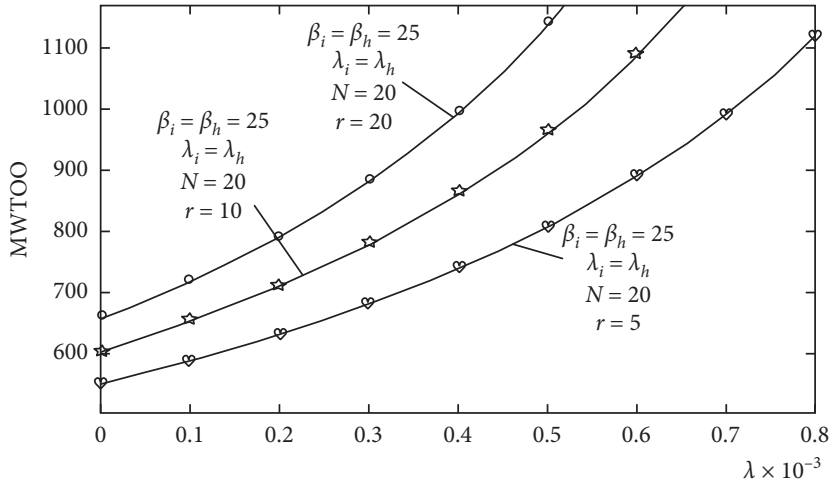

(c)

Figure 6: The change trend of MWTOO. (a) Range of MWTOO with $\lambda^{\prime}$ s change under different $N$. (b) Range of MWTOO with $\lambda^{\prime}$ s change under different $\beta$. (c) Range of MWTOO with $\lambda^{\prime}$ s change under different $r$. 
which shows that the new model can settle the priority challenge of VIP orders without overextending the waiting time for ordinary users, which is significant in maintaining customer loyalty for logistics enterprises.

\section{Conclusions}

In this study, we apply a polling system to analyse a DPS model considering priority orders. First, we divide the arrival orders into ordinary orders and priority orders. Priority orders refer to those orders that need priority processing, and ordinary orders refer to those orders that need not be processed immediately and have loose time windows. Correspondingly, we divide the picking machines into priority picking machines, under the 1-limited control strategy, and ordinary picking machines, under the exhaustive control strategy. A probability generating function is then established to analyse the system.

Our study extends the method and theory of the polling system in a dynamic order-picking model and contributes to the existing research in two aspects: First, the prior studies $[11,13]$ did not consider a situation in the presence of priority orders by using the polling system in picking models. However, we propose a polling-based picking model that not only emphasizes the service quality for VIP orders but also takes into account the efficiency in servicing ordinary orders. Second, we propose an "exhaustive parallel 1limited" based dynamic order-picking model that combines the polling system, the probability generating function approach, and the Markov chain. The numerical analysis results reveal the practicality and effectiveness of the proposed approach.

In the end, this study has a number of limitations. For instance, there are various constraints for the proposed picking system, for example, the constraints of the order arrival process, the picking process, and the picking machine switching process. The generating function also changes correspondingly in line with the constraint change. Therefore, this may cause the problem-solving methods to be more complicated and cannot offer an effective solution. Hence, future work should pay more attention to relax the proposed polling picking system's constraints so that it is more consistent with the actual situation. In addition, with regard to the proposed polling-based model, we use an approximate calculation and simulation experiment method to compute the system characteristic parameters, which may bring some inaccuracy to the order-picking result. At present, the common methods for solving the polling-based model are the buffer occupancy method, station-time method, descendant set method, embedded Markov chain method, mean value analysis method, and computer simulation method [49-51]. However, these methods can only solve the general polling-based model, such as the first- and second-order characteristic parameters. For the third-order characteristic parameters, due to the complexity of the calculation, only approximate solutions are obtained. Future research may consider focusing on the solution methods of the complex polling- based model to improve the calculation precision of the polling-based system parameters.

\section{Data Availability}

All relevant data are provided within the article.

\section{Conflicts of Interest}

The authors declare that there are no conflicts of interest regarding the publication of this paper.

\section{Acknowledgments}

This study was supported by the National Natural Science Foundation of China under project nos. 71661029, 71862035 , and 71502159 and the Yunnan Fundamental Research Project under grant no. 2019FB085.

\section{References}

[1] Y. S. Cheung, "Joint polling and contention based feedback algorithm to exploit multiuser diversity," Journal of Communications, vol. 4, no. 5, pp. 348-356, 2009.

[2] J. M. Tarn, M. A. Razi, and H. J. Wen, "E-fulfillment: the strategy and operational requirementsfillment: the strategy and operational requirements," Logistics Information Management, vol. 16, no. 5, pp. 350-362, 2003.

[3] S. Perez and P. D. Wright, "In-store pickup and returns for a dual channel retailer," Ieee Transactions on Engineering Management, vol. 64, no. 4, pp. 491-504, 2017.

[4] S. Moons, K. Ramaekers, A. Caris, and Y. Arda, "Integration of order picking and vehicle routing in a B2C e-commerce context," Flexible Services and Manufacturing Journal, vol. 30, no. 4, pp. 813-843, 2018.

[5] J. I. U. Rubrico, T. Higashi, H. Ota, and J. Otad, "Online rescheduling of multiple picking agents for warehouse management," Robotics and Computer-Integrated Manufacturing, vol. 27, no. 1, pp. 62-71, 2011.

[6] E. Ardjmand, H. Shakeri, M. Singh, and O. Sanei Bajgiran, "Minimizing order picking makespan with multiple pickers in a wave picking warehouse," International Journal of Production Economics, vol. 206, pp. 169-183, 2018.

[7] G. Baruffaldi, R. Accorsi, and R. Manzini, "Warehouse management system customization and information availability in 3pl companies," Industrial Management \& Data Systems, vol. 119, no. 2, pp. 251-273, 2019.

[8] J. J. Bartholdi and S. T. Hackman, "Warehouse \& distribution science release," Industrial Management \& Data Systems, vol. 94, 2011.

[9] D. Fussler and N. Boysen, "Efficient order processing in an inverse order picking system," Computers \& Operations Research, vol. 88, pp. 150-160, 2017.

[10] Y.-C. Ho and J.-W. Lin, "Improving order-picking performance by converting a sequential zone-picking line into a zone-picking network," Computers \& Industrial Engineering, vol. 113, pp. 241-255, 2017.

[11] Y. Gong and R. De Koster, "A polling-based dynamic order picking system for online retailers," IIE Transactions, vol. 40, no. 11, pp. 1070-1082, 2008.

[12] J. R. Otto and Q. B. Chung, "A framework for cyber-enhanced retailing: integrating E-commerce retailing with brick-and- 
mortar retailing," Electronic Markets, vol. 10, no. 3, pp. 185191, 2000.

[13] Y. Gong, E. Winands, and R. D. Koster, "A real-time picking and sorting system in e-commerce distribution centers," Electronic Markets, vol. 10, 2012.

[14] P. J. Xu, R. Allgor, and S. C. Graves, "Benefits of Reevaluating Real-Time Order Fulfillment Decisionsfits of reevaluating real-time order fulfillment decisions," Manufacturing \& Service Operations Management, vol. 11, no. 2, pp. 340-355, 2009.

[15] B.-I. Kim, S. S. Heragu, R. J. Graves, and A. Onge, "Realization of a short cycle time in warehouse replenishment and order picking," International Journal of Production Research, vol. 41, no. 2, pp. 349-364, 2003.

[16] T. Le-Duc and R. M. B. M. de Koster, "Travel time estimation and order batching in a 2-block warehouse," European Journal of Operational Research, vol. 176, no. 1, pp. 374-388, 2007.

[17] D. Claeys, I. Adan, and O. Boxma, "Stochastic bounds for order flow times in parts-to-picker warehouses with remotely located order-picking workstations," European Journal of Operational Research, vol. 254, no. 3, pp. 895-906, 2016.

[18] R. De Koster, T. Le-Duc, and K. J. Roodbergen, "Design and control of warehouse order picking: a literature review," European Journal of Operational Research, vol. 182, no. 2, pp. 481-501, 2007.

[19] J. Gu, M. Goetschalckx, and L. F. Mcginnis, "Research on warehouse design and performance evaluation: a comprehensive review," European Journal of Operational Research, vol. 203, no. 3, pp. 539-549, 2010.

[20] W. Lu, D. Mcfarlane, V. Giannikas, and Q. Zhang, "An algorithm for dynamic order-picking in warehouse operations," European Journal of Operational Research, vol. 203, 2015.

[21] R. De Koster, "Performance approximation of pick-to-belt orderpicking systems," European Journal of Operational Research, vol. 72, no. 3, pp. 558-573, 1994.

[22] J. C.-H. Pan and M.-H. Wu, "A study of storage assignment problem for an order picking line in a pick-and-pass warehousing system," Computers \& Industrial Engineering, vol. 57, no. 1, pp. 261-268, 2009.

[23] R. Manzini, R. Accorsi, M. Gamberi, and S. Penazzi, "Modeling class-based storage assignment over life cycle picking patterns," International Journal of Production Economics, vol. 203, 2015.

[24] E. Atmaca and A. Ozturk, "Defining order picking policy: a storage assignment model and a simulated annealing solution in AS/RS systems," Applied Mathematical Modelling, vol. 37, no. 7, pp. 5069-5079, 2013.

[25] D. Battini, M. Calzavara, A. Persona, and F. Sgarbossa, “Order picking system design: the storage assignment and travel distance estimation (SA\&TDE) joint method," International Journal of Production Research, vol. 53, no. 4, pp. 1077-1093, 2015.

[26] F. Guerriero, O. Pisacane, and F. Rende, "Comparing heuristics for the product allocation problem in multi-level warehouses under compatibility constraints," Applied Mathematical Modelling, vol. 39, no. 23-24, pp. 7375-7389, 2015.

[27] J. Won and S. Olafsson, "Joint order batching and order picking in warehouse operations," International Journal of Production Research, vol. 43, no. 7, pp. 1427-1442, 2005.

[28] L. Tang and H. Gong, "A hybrid two-stage transportation and batch scheduling problem," Applied Mathematical Modelling, vol. 32, no. 12, pp. 2467-2479, 2008.
[29] R. W. Grubbström, "Dynamic lotsizing with a finite production rate," International Journal of Production Economics, vol. 149, no. 3, pp. 68-79, 2014.

[30] V. I. Nieuwenhuyse and R. B. M. D. Koster, "Evaluating order throughput time in 2-block warehouses with time window batching," International Journal of Production Economics, vol. 121, no. 2, pp. 654-664, 2009.

[31] C. Theys, O. Bräysy, W. Dullaert, and B. Raa, "Using a TSP heuristic for routing order pickers in warehouses," European Journal of Operational Research, vol. 200, no. 3, pp. 755-763, 2010.

[32] S. Rath and W. J. Gutjahr, "A math-heuristic for the warehouse location-routing problem in disaster relief," Computers \& Operations Research, vol. 42, pp. 25-39, 2014.

[33] C. Chackelson, A. Errasti, D. Ciprés, and F. Lahoz, "Evaluating order picking performance trade-offs by configuring main operating strategies in a retail distributor: a Design of Experiments approach," International Journal of Production Research, vol. 51, no. 20, pp. 6097-6109, 2013.

[34] E. H. Grosse and C. H. Glock, "The effect of worker learning on manual order picking processes," International Journal of Production Economics, vol. 51, 2014.

[35] S. Hong, "Two-worker blocking congestion model with walk speed $\mathrm{m}$ in a no-passing circular passage system," European Journal of Operational Research, vol. 235, no. 3, pp. 687-696, 2014.

[36] P. H. P. Yeow and D. T. Goomas, "Ergonomics improvement in order selection in a refrigerated environment," Human Factors \& Ergonomics in Manufacturing \& Service Industries, vol. 24, no. 3, pp. 262-274, 2014.

[37] T. Van Gils, A. Caris, K. Ramaekers, K. Braekers, and R. B. M. De Koster, "Designing efficient order picking systems: the effect of real-life features on the relationship among planning problems," Transportation Research Part E-Logistics and Transportation Review, vol. 125, pp. 47-73, 2019.

[38] E. H. Grosse, C. H. Glock, and M. Y. Jaber, "The effect of worker learning and forgetting on storage reassignment decisions in order picking systems," Computers \& Industrial Engineering, vol. 66, no. 4, pp. 653-662, 2013.

[39] E. H. Grosse, C. H. Glock, M. Y. Jaber, and W. P. Neumann, "Incorporating human factors in order picking planning models: framework and research opportunities," International Journal of Production Research, vol. 53, no. 3, pp. 695-717, 2014.

[40] M. M. Srinivasan, "Nondeterministic polling systems," Management Science, vol. 37, no. 6, pp. 667-681, 1991.

[41] Y. A. Bozer and J. H. Park, "Expectedmove request waiting times in single-device, polling-basedmaterial handling systems," IIE Transactions, vol. 33, no. 5, pp. 55-63, 1999.

[42] D. Xu, N. Shi, and R. K. Cheung, "Heavy traffic analysis of a single vehicle loop in an automated storage and retrieval systemfic analysis of a single vehicle loop in an automated storage and retrieval system," OR Spectrum, vol. 29, no. 3, pp. 489-512, 2007.

[43] P. Piccinini, R. Gamberini, A. Prati, B. Rimini, and R. Cucchiara, "An automated picking workstation for healthcare applications," Computers \& Industrial Engineering, vol. 64, no. 2, pp. 653-668, 2013.

[44] I. Eliazar, "Gated polling systems with Levy inflow and interdependent switchover times: a dynamical-systems approach," Queueing Systems, vol. 49, no. 1, pp. 49-72, 2005.

[45] K. K. Leung, "Cyclic-service systems with nonpreemptive, time-limited service," IEEE Transactions on Communications, vol. 42, no. 8, pp. 2521-2524, 1994. 
[46] O. Boxma, J. Bruin, and B. Fralix, "Sojourn times in polling systems with various service disciplines," Performance Evaluation, vol. 66, no. 5, pp. 621-639, 2009.

[47] H. Takagi, "Queuing analysis of polling models," ACM Computing Surveys (CSUR), vol. 20, no. 1, pp. 5-28, 1988.

[48] H. Takagi, "Mean message waiting times in symmetric multiqueue systems with cyclic service," Performance Evaluation, vol. 5, no. 4, pp. 271-277, 1985.

[49] N. Chernova, S. Foss, and B. Kim, "A polling system whose stability region depends on the whole distribution of service times," Operations Research Letters, vol. 41, no. 2, pp. 188-190, 2013.

[50] J.-P. L. Dorsman, S. C. Borst, O. J. Boxma, and M. Vlasiou, "Markovian polling systems with an application to wireless random-access networks," Performance Evaluation, vol. 41, 2015.

[51] Z. Liu, Y. Chu, and J. Wu, "Heavy-traffic asymptotics of a priority polling system with threshold service policy," Computers \& Operations Research, vol. 65, pp. 19-28, 2016. 